\section{Abdulkarim A Johnson A Yahaya-Kongoila S Mohammed SS}

\title{
Measles: the past, the present and the future
}

DOI:http://dx.doi.org/10.4314/njp.v39i3,12

Accepted : 27th February 2012

Abdulkarim A ( $\square)$

Johnson A, Yahaya-Kongoila S

Mohammed SS

Department of Paediatrics and

Child Health,

University of Ilorin Teaching

Hospital,

Ilorin, Nigeria

\begin{abstract}
Measles is one of the most infectious diseases known to affect man. It spreads rapidly from an index case to many susceptible individuals resulting in cycles of epidemics in different parts of the world especially in the African continent. The contribution of measles to childhood mortality and morbidity remains high because it gives rise to complications such as pneumonia, malnutrition and blindness. Measles vaccine is known to be efficacious and effective; however appearance of epidemics in parts of Europe and the USA in recent times underscores the fact that low vaccine uptake will always leave a large number of susceptible indi-
\end{abstract}

viduals with a potential for epidemics. The history, aetiopathogenesis, clinical features (including complications), diagnostic tools, treatment and prevention are discussed in this article. The most important steps to prevent measles cases and deaths would be to institutionalize the two dose measles vaccine regime; give supplemental Vitamin A; strengthen health systems to be able to adequately deliver the vaccine and supplement, carry out surveillance and offer treatment for cases. There is no doubt that global partnerships remain relevant in order to consolidate and accelerate prevention of measles, thus, they must continue to be supported.

\section{Introduction}

Reports of measles go back to at least 700BC. However, the first scientific description of the disease and its distinction from smallpox attributed to the Muslim physician Ibn Razi(Rhazes) 860-932 who published a book entitled "Smallpox and Measles" (in Arabic: Kitab fi aljadari wa-al-hasbah).

\section{The Facts}

Measles remains a leading cause of death among young children globally, despite the availability of a safe and effective vaccine. An estimated 197000 people died from measles in 2007, mostly children under the age of five. In developing countries, measles affects 30 million children a year and causes 1 million deaths. Measles causes 15,000-60,000 cases of blindness per year. Approximately 30 million measles cases are reported annually. Most reported cases are from Africa. In 1998, the cases of measles per 100,000 total population reported to the World Health Organization was 1.6 in the Americas, 8.2 in Europe, 11.1 in the Eastern Mediterranean region, 4.2 in South East Asia, 5.0 in the Western Pacific region, and 61.7 in Africa. Only 187 confirmed cases were reported in the Western Hemisphere (mainly in Venezuela, Mexico, and the United States) (Fig 1-5)

Fig 1

\section{Measles global annual reported cases and MCV coverage, 1980-2009}

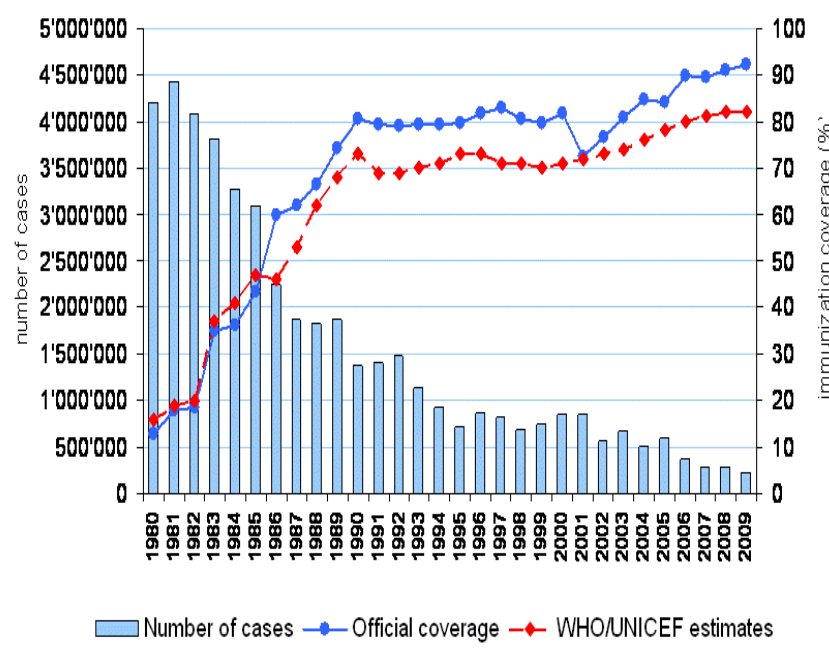




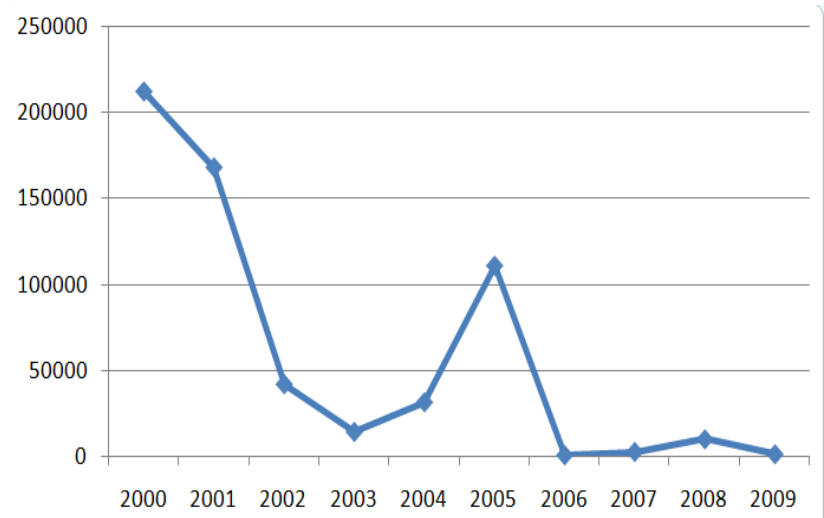

Fig 2 : Nigerian incidence series over 10 years (Source: WHO)

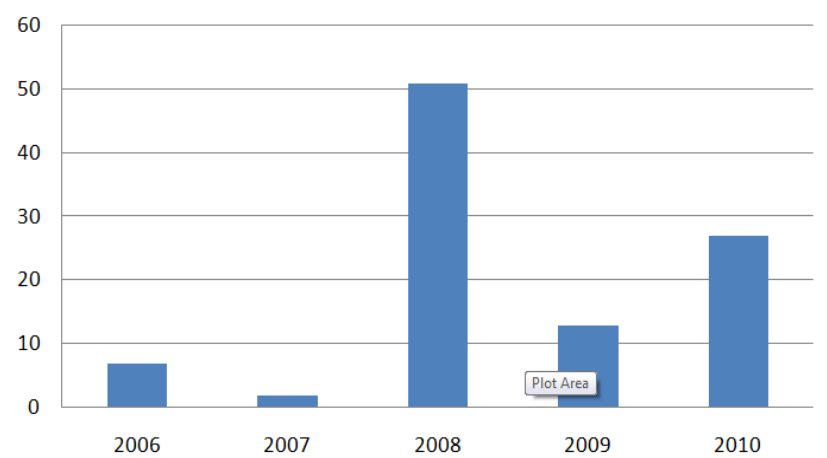

Fig 3 : A 5-year measles admission in EPU, UITH

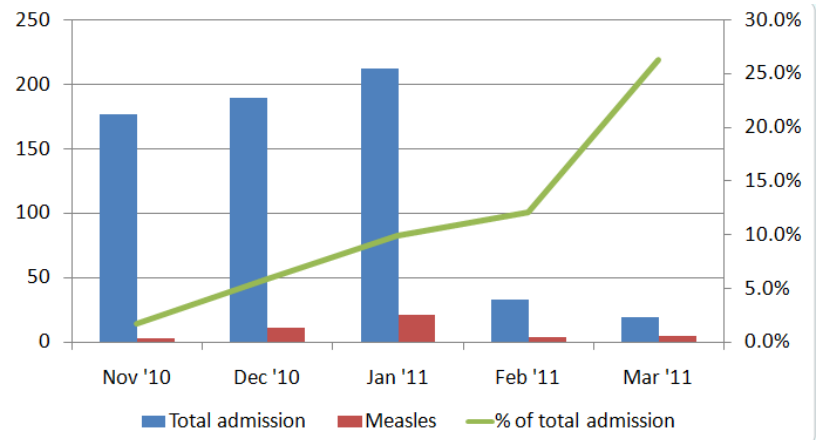

Fig 4: EPU admission in the last 5 months (as at 7th March, 2011)

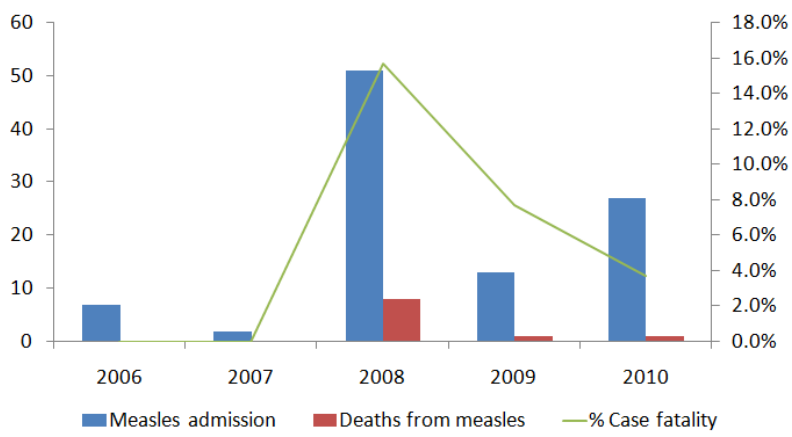

Fig 5: Deaths from Measles admitted in EPU over 5 years

\section{Measles}

It is an infection of the respiratory system caused by a virus, specifically Paramyxoviruses of the genus Morbil- livirus Morbilliviruses, like other paramyxoviruses, are enveloped, single-stranded, negative-sense RNA viruses. The measles virus is a spherical, non segmented, single-stranded RNA virus in the Morbillivirus family, closely related to the rinderpest and canine distemper viruses. It contains six structural proteins, three that are complexed to the RNA and three that are associated with the viral membrane envelope. The $\mathrm{F}$ (fusion) protein is responsible for fusion of virus and host cell membranes, viral penetration and hemolysis. The $\mathrm{H}$ (hemagglutinin) protein is responsible for adsorption of the virus to cells. There is only one serotype of Measles virus and no subtypes have yet been recognized

\section{Pathogenesis}

The essential lesion of measles is found in the skin, conjunctivae, and mucous membranes of nasopharynx, bronchi, and intestinal tract. Serous exudate and proliferation of mononuclear cells and a few polymorphonuclear cells occur around the capillaries. Hyperplasia of lymphoid tissue usually occurs particularly in the appendix. The pathologic lesion is the Warthin-Finkeldey giant cell. These are multinucleated reticuloendothelial giant cells of up to 100 micrometers in diameter.

In the skin, this reaction can be noted around the sebaceous glands and hair follicles. Koplik spots consist of serous exudate and proliferation of endothelial cells similar to those seen in the skin lesions.

\section{Transmission}

Measles is spread through respiration (contact with fluids from an infected person's nose and mouth, either directly or through aerosol transmission), and is highly contagious - $90 \%$ of people without immunity sharing a house with an infected person will catch it. The infection has an average incubation period of 14 days (range 6-19 days). Infectivity lasts from 2-4 days prior to 2-5 days following the onset of the rash. Replication occurs in nasopharynx and regional lymph nodes. Primary viraemia occurs 2-3 days after exposure while secondary viremia occurs 5-7 days after exposure with spread to tissues.

\section{Clinical Features}

Incubation period ranges from 6-19 days. It has a prodromal period which presents with stepwise increase in fever to $39.4^{0} \mathrm{C}$ or higher, cough, coryza, conjunctivitis and Koplik spots (rash on mucous membranes).

The rash occurs 2-4 days after prodrome and 14 days after exposure. It is maculopapular and later become confluent beginning on face and head. It persists 5-6 days and fades in order of appearance. Patients are contagious from 1-2 days before onset of symptoms. Healthy children are also contagious during the period from 3-5 days before the appearance of the rash to 4 days after the onset of rash. On the other hand, immunocompromised individuals can be contagious during the entire duration of the illness. 


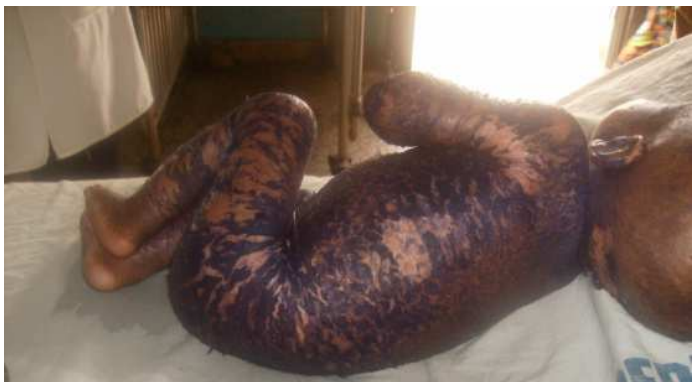

Risk factors for infection

Risk of infection is increased among children with immunodeficiency due to HIV or acquired immunodeficiency syndrome (AIDS), leukemia, alkylating agents, or corticosteroid therapy, regardless of immunization status. Also those that travel to areas where measles is endemic or contact with travellers to endemic areas, infants who lose passive antibody prior to the age of routine immunization, children with malnutrition, underlying immunodeficiency and vitamin A deficiency are at increased risk of contacting measles.

\section{Mortality Rate in Measles}

The mortality rate associated with uncomplicated measles in immunocompetent, well nourished children is low but is much higher in malnutrition (marked in African children), in immunocompromised, and to lesser extent with age.

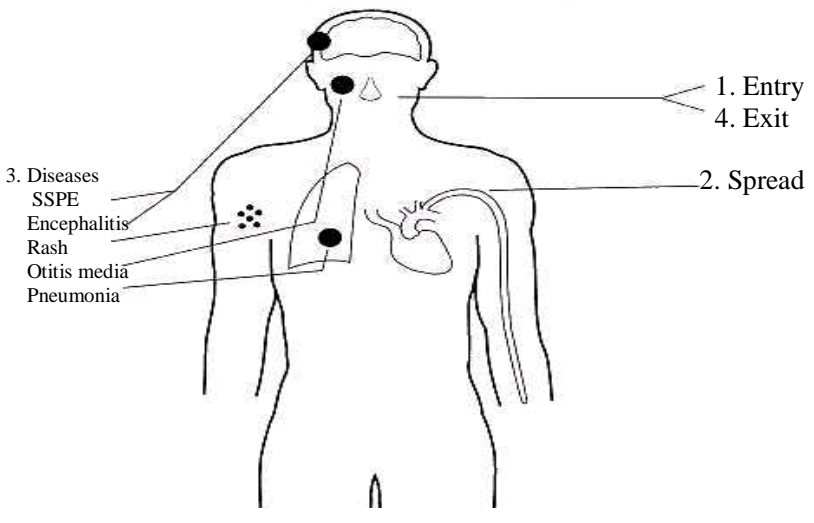

Prominent features in Measles

\section{Modified Measles}

Modified measles occurs in children who have received serum immunoglobulin after their exposure to measles. The measles symptom complex may still occur, but the incubation period is as long as 21 days, with the same symptoms as measles but milder.

\section{Atypical Measles}

When exposed to the measles virus, a mild or nonexistent prodrome of fever, headache, abdominal pain, and myalgias precedes a rash that begins on the hands and feet and spreads centrally. The rash is most prominent in the body creases and may be macular, hemorrhagic vesicles, petechial, or urticarial. Complications may include pneumonia, pleural effusion, hilar lymphadenopathy, Hepatosplenomegaly, hyperesthesia, or paresthesia. Atypical measles occurs in individuals who were previously immunized with the killed measles vaccine between 1963 and 1967 and who have incomplete immunity.

\section{Subacute sclerosing panencephalitis (SSPE)}

It is a neurodegenerative disease caused by persistent infection of the brain by an altered form of the measles virus. Neither the biology underlying the viral persistence nor the triggering mechanism for viral reactivation is well understood. In most cases, infected children remain symptom-free for 6-15 years after acute measles infection. Subacute sclerosing panencephalitis (SSPE) is rare chronic, progressive encephalitis that affects primarily children and young adults, caused by a persistent infection of immune resistant measles virus (which can be a result of a mutation of the virus itself). 1 in 100,000 people infected with measles develop SSPE. SSPE is 'incurable' but the condition can be managed by medication if treatment is started at an early stage.

\section{Clinical Presentation of SSPE}

Characterized by a history of primary measles infection usually before the age of 2 years, followed by several asymptomatic years (6-15 on average), and then gradual, progressive psycho neurological deterioration, consisting of personality change, seizures, myoclonus, ataxia, photosensitivity, ocular abnormalities, spasticity, and coma.

\section{Diagnosis of Measles}

Most cases of Measles are diagnosed clinically, usually in patient's home or in General practice. Direct Virological confirmation is difficult in most of the developing countries.

\section{Diagnosis by Microscopy}

Production of multinucleate giant cells with inclusion bodies is path gnomonic for measles. During the prodromal phase, such cells are detectable in the NPS (nasopharyngeal secretions). This is more rapid and practical than virus isolation.

\section{Diagnosis with Immunofluorescence}

Direct and indirect immunofluorescence has been used extensively to demonstrate MV antigens in cells from NPS specimens. This technique can also be applied to the urine as such cells may be present in the urine 2 to 5 days after the appearance of the rash. 
Diagnosis by Viral Isolation

Measles virus can be isolated from a variety of sources, e.g. throat or conjunctival washings, sputum, urinary sediment cells and lymphocytes. Primary human kidney (HEK) cells are the best, although primary monkey kidney can be used as well. Continuous cell lines such as Vero cells can also be used although they are not as efficient as primary cell lines.

\section{Diagnosis by Serology}

Diagnosis of measles infection can be made if the antibody titres rise by 4 fold between the acute and the convalescent phase or if measles-specific $\operatorname{IgM}$ is found. The methods that can be used include HAI, CF, neutralization and ELISA tests.

\section{Differential diagnosis of measles}

1. Rubella

2. Scarlet fever

3. Infectious mononucleosis, erythema infectiosum, echovirus and coxsackievirus infections

4. Drug rashes (eg, from phenobarbital or sulfonamides

How to distinguish measles from some other conditions

- Rubella: A recognizable prodrome is absent, fever and other constitutional symptoms are absent or less severe, postauricular and suboccipital lymph nodes are enlarged (and usually tender), and duration is short.

- Roseola infantum: The rash resembles that of measles, but it seldom occurs in children $>3 \mathrm{yr}$. Initial temperature is usually high, Koplik's spots and malaise are absent, and defervescence and rash occur simultaneously.

- Drug rashes: A drug rash often resembles the measles rash, but a prodrome is absent, there is no cephalocaudal progression or cough, and there is usually a history of recent drug exposure

\section{Complications}

Some complications include; pneumonia, croup, pneumothorax, bacterial superinfection, acute thrombocytopenic purpura, post-measles anergy, encephalitis, subacute sclerosing panencephalitis and atypical measles syndrome

\section{Treatment}

Severe complications from measles can be avoided though supportive care that ensures good nutrition, adequate fluid intake and treatment of dehydration with WHO-recommended oral rehydration solution (to replace fluids and other essential elements lost from diarrhoea or vomiting). Antibiotics should be prescribed to treat eye and ear infections, and pneumonia.

\section{Treatment options in Developing Countries}

All children in developing countries diagnosed with measles should receive two doses of vitamin A supplements, given 24 hours apart. This can help prevent eye damage and blindness. Vitamin A supplements have been shown to reduce the number of deaths from measles by $50 \%$.

\section{Vaccination}

The Vaccines are live attenuated containing Edmonston B or Schwartz strains which will give seroconversion rate of $90 \%$. The immunity produced may be lifelong.

\section{Changing trends for a Booster Dose}

In 2007 , about $82 \%$ of the world's children received one dose of measles vaccine by their first birthday through routine health services, up from $72 \%$ in 2000 . (Two doses of the vaccine are recommended to ensure immunity, as it has been found that about $15 \%$ of vaccinated children fail to develop immunity from the first dose).

\section{Two doses of Measles Vaccine}

Continued progress depends on ensuring that all children receive two doses of measles vaccine including one dose by their first birthday, strengthening disease surveillance systems, and providing effective treatment for measles. However, in Nigeria, this two-dose vaccine policy has not yet been adopted.

\section{Global Initiatives}

The Measles Initiative - a collaborative effort of WHO, UNICEF, the American Red Cross, the United States Centres for Disease Control and Prevention, and the United Nations Foundation - and other public and private partners play key roles in advancing the global measles strategy

\section{WHO and UNICEF are collaborating to ensure;}

- Strong routine immunization: for children by their first birthday.

- A 'second opportunity' for measles immunization through mass vaccination campaigns, to ensure that all children receive at least one dose.

- Effective surveillance in all countries to quickly recognize and respond to measles outbreaks.

- Better treatment of measles cases, to include vitamin A supplements, antibiotics if needed, and supportive care that prevents complications.

\section{Acknowledgement}

Reproduced with kind permission of the department of Paediatrics and Child Health of the University of Ilorin Teaching Hospital, Ilorin Nigeria owners of the Ilorin Paediatric Digest 2011. 


\section{References}

1. Behrman,RE Kliegman RM, Jenson HB. (Eds) Nelson's textbook of Paediatrics 17th Ed. 2004 Elsevier.
2. Rao, TV. Measles Update: Created for medical and paramedical students in a developing world.
3. Measles and measles vaccine. Epidemiology and prevention of vaccine-preventable diseases. National Center for immunisation and respiratory diseases. Center for Disease control and prevention Revised May 2009. 